\title{
Enhanced tumor-infiltrating lymphocytes (eTIL) for cellular therapy of patients with pancreatic cancer or glioblastoma
}

\author{
Qingda Meng, Zhenjiang Liu, Markus Maeurer*, Elena Rangelova, Aditya Ambati, Thomas Poiret, Lalit Rane, \\ Shanshan Xie, Bartek Jiri, Caroline Verbeke, Oscar Persson, Matthias Löhr, Ralf Segersvärd, Ernest Dodoo
}

From 30th Annual Meeting and Associated Programs of the Society for Immunotherapy of Cancer (SITC 2015) National Harbor, MD, USA. 4-8 November 2015

\section{Purpose}

The generation of $\mathrm{T}$ lymphocytes with specific reactivity against autologous tumor cells is a prerequisite for effective targeted cellular therapies. We established a protocol for tumor infiltrating lymphocytes (TILs) cultures from small biopsies or surgically resected material obtained from patients with pancreatic ductal adenocarcinoma (PDAC) and patients with glioblastoma.

\section{Methods}

Tumor specimens obtained from 17 patients with PDAC and 16 patients with glioma were cultured with TIL medium, containing IL-2, IL-15 and IL-21. Activity of TILs directed against shared tumor associated antigens (TAAs, mesothelin, survivin and NY-ESO-1, EGFRvIII) was detected by Intracellular staining (ICS). Cytotoxicity was measured using a Chromium-51 release assay and the specific activity of TILs against autologous tumor was measured by INF- $\gamma$ production. TCRVb analysis was investigated by a multiplex antibody TCR Vb specific panel. TCR clonality was gauged by CDR3 region PCR analysis and subsequent sequencing. The $\mathrm{T}$ cell phenotype, as well as activation/exhaustion marker profile was tested by flow cytometry.

\section{Results}

We could reliably expand TILs, that resided predominantly in the central memory population (CD45RA', $\mathrm{CCR}^{+}$), from $33 / 33$ patients. We were able to expand about $1.5 \times 10 \mathrm{e} 9$ TIL from small PDAC biopsies. TIL showed reactivity to common TAAs, i.e. to mesothelin (16/17 in PDAC, 8/16 in glioma), survivin (12/17 in
PDAC, $6 / 16$ in glioma) or to NY-ESO-1(11/17 in PDAC, 6/16 in glioma) defined by intracellular cytokine staining. TIL cultures exhibited preferential usage of V $\beta$ families (e.g. some TIL showed 99.3\% Vb13.2, 99.60\% $\mathrm{V} \beta 2,97.00 \% \mathrm{~V} \beta 5.1$ in $\mathrm{CD}^{+}$or $\left.\mathrm{CD} 8^{+} \mathrm{TIL}\right)$. A PDAC Vb13.2 ${ }^{+}, \mathrm{CD}^{+}$TIL clone strongly recognized autologous tumor cells, defined by INF- $\gamma$ production, which could be blocked by the anti-HLA class 1 antibody (W6/32). TIL from glioma patients exhibited up to $25 \%$ IFN- $\gamma$ and TNF- $\alpha$ production directed against autologous tumor cells, defined by ICS. TILs from PDAC and glioma showed strong cytolytic functions directed against autologous tumor cells, i.e. up to $70 \%$ specific lysis at an effector/target ratio at $25 / 1$ by chromium- 51 release assay.

\section{Conclusions}

We have optimized methods for generating pancreatic cancer and glioblastoma specific TIL cultures from small resected tumor specimens. Tumor specimens are currently sequenced to detect potential targets for antiPDAC and anti-glioma directed T cell clones. A Phase I study to administer TIL for patients with pancreatic cancer or glioblastoma will start at Karolinska.

Published: 4 November 2015

doi:10.1186/2051-1426-3-S2-P35

Cite this article as: Meng et al.: Enhanced tumor-infiltrating

lymphocytes (eTIL) for cellular therapy of patients with pancreatic cancer or glioblastoma. Journal for ImmunoTherapy of Cancer 2015 3(Suppl 2):P35.

Karolinska Institutet, Stockholm, Sweden 